\title{
Sintering and additive manufacturing: "additive manufacturing and the new paradigm for the jewellery manufacturer"
}

\author{
Frank Cooper ${ }^{1}$ (D)
}

Received: 26 May 2015/ Accepted: 3 December 2015/Published online: 15 January 2016

(C) Springer International Publishing Switzerland 2016

\begin{abstract}
The use of various sintering technologies, allied to suitable powder metallurgy, has long been the subject of discussion within the global jewellery manufacturing community. This exciting, once theoretical and experimental technology is now undoubtedly a practical application suitable for use by the jewellery industry. All parts of the jewellery industry supply and value chains, and especially design and manufacturing, now need to become aware very quickly of just how unsettling and disruptive this technology introduction has the potential to become. This paper will offer various viewpoints that consider not only the technology and its application to jewellery manufacture but will also consider the new design potentials of the technology to the jewellery industry. It will also briefly consider how that design potential is being taught to future generations of jewellery designers at the Birmingham School of Jewellery. We shall also discuss in some detail the economics of and potential for new and different business models that this technological paradigm might offer the global jewellery industry.
\end{abstract}

Keywords Additive manufacturing - Direct metal laser melting · Direct metal laser sintering · Jewellery · Precious metals $\cdot \mathrm{CAD} \cdot 3 \mathrm{D}$ printing

Frank Cooper

frank.cooper@bcu.ac.uk

1 Jewellery Industry Innovation Centre, School of Jewellery, Birmingham City University, 85-87 Vittoria Street, Hockley, Birmingham B1 3PA, UK

\section{Rationale}

This paper intends to explore and open up for debate by the jewellery industry what actions and understanding might be required in order to facilitate the transfer and acceptance of precious metal direct metal laser melting (DMLM) technologies and processes into a manufacturing process specifically tailored for the jewellery manufacturing industries and their related value and supply chains. The goal of the Jewellery Industry Innovation Centre (JIIC) and its parent institution, the Birmingham School of Jewellery, $\mathrm{UK}$, is to encourage its students to develop, design and produce computer aided design (CAD) examples of jewellery products to challenge, prove, and democratise the processes and materials required for the application of precious metal DMLM technology into the production facilities of small and medium-sized enterprises (SMEs) within the UK jewellery manufacturing sector. The paper also assesses and attempts to quantify the current perceived industry needs for an adaptable, low-volume and innovative new technology that will facilitate rapid responses by SMEs to the consumer's demands for more custom-made, individually designed and personalised jewellery products. Typical jewellery manufacturing processes like lost-wax investment casting or stamping do not have either the necessary quick response times or, more importantly, the design and production flexibility required to address these issues. This paper is intended to help increase jewellery industry awareness, knowledge, and especially it is hoped to speed up jewellery industry uptake of the new design and production capabilities offered by the DMLM processes for working with precious metals. In order to aid the reader who would like to drill down deeper into some of the key technical details of this technology, especially in the areas of parameters, pre and post-processing, have been 
referenced. Most of these references relate to papers presented at symposia and congresses that have addressed these specific areas of explicit research.

\section{The economic argument}

This section will focus primarily on the European jewellery market sector and its financial models.

The European Union (EU) has traditionally been an important supplier of high-quality jewellery to the world's markets, and is also considered to be the second or third largest market for jewellery consumption, after the USA (China and India vie for the other places depending on the statistical analysis method used). Sales of jewellery in most EU countries are thought to have risen steadily in the decade from 2005 to the present; however, this volume market is predominantly supplied with jewellery items manufactured outside the EU. Socio-economic factors mean that it is very difficult for the European jewellery manufacturing industry to be competitive on price alone. Recent global, and particularly EU, economic and financial crises have further impacted the EU jewellery manufacturing industry and recent price fluctuations in all precious metals have somewhat weakened jewellery sales. Consumers seem to have reduced their expenditure on jewellery and sought personalised pieces with greater associated personal value [1]. High-quality jewellery manufacturing has long been an important sector within the EU economy. Detailed information on employment statistics for the EU jewellery industry is difficult to source and define accurately but there are thought to be some 30,000 smaller companies, with less than 250 employees each, and around 200 larger companies with an estimated total of 180,000 employees. These are companies that specialise either in a style of jewellery design or in a stage of production [2]. The World Gold Council has estimated that in 2014 the global demand for gold jewellery was US $\$ 100$ billion [3]. Within the EU and other developed economies, consumers have been educated by the fast-moving, digital, online revolution to expect a continuous and regularly updated choice of new and innovative products. This has impacted consumer-buying patterns, resulting in a surfeit of choice and an ever-increasing competition for their disposable incomes. It could safely be predicted that the future high-value jewellery market could well be increasingly driven by a growing demand for custom-made, personalised, individually designed and innovative designs of high-quality and high-value jewellery as high precious metal prices have resulted in many consumers now considering the design and innovation within a jewellery item as equal to if not more important than its base intrinsic value [4]. Increased affluence in the newly emerging economies, coupled with a rising number of marriages, working women, increased shopping opportunities and an online interest in fashion, are thought to be the main driving forces behind the latest growth in precious metal jewellery sales in these areas. However, consumers are also more careful with their spending. There is considered to be a growing fatigue towards 'fast turnover fashions' and many consumers now have increasing opportunity to favour good and uniquely personalised 'statement' designs and regard these as more important than the intrinsic value of a jewellery piece. Global economic uncertainty makes it difficult to precisely forecast future jewellery industry trends, but the market is predicted in some quarters to begin to expand. In the near future the EU jewellery market is expected to grow, especially in the Eastern EU and accession countries due in part to their newly emerging middle classes. It is the contention of this paper that the EU market in particular will increasingly demand higher quality products, coupled with original designs and statement jewellery with added perceived value, personalisation, or new production technologies [5-8]. A key question is therefore: "Is there a viable economic argument for considering adoption of DMLM technology?" The author believes that the jewellery-manufacturing sector in the EU has the potential to grow significantly further if new hightechnology approaches such as DMLM are adopted and exploited effectively. While all materials used in the production of precious metal jewellery are intrinsically expensive, DMLM offers access to a technology shift that is able to potentially reduce material usage while offering new market opportunities.

\section{Technology and design}

It is widely accepted that DMLM was developed during the 1990s in Germany [9, 10]. Beginning with CAD data [11], several layers of metallic powder are successively deposited one on top of the other. Each layer of powder is heated using a focused laser beam corresponding to a selected cross-section of the part to be produced. The powder bed is then dropped incrementally and another layer of powder is applied and smoothed by a re-coating blade prior to application of the next pass of the laser beam, simultaneously fusing each new layer of powder to the layer below it. The method differs from the related technique of direct metal laser sintering (DMLS) in that the layer of metallic powder is fully molten throughout. The method does not require any binders or fluxing agents. Each pass of the laser beam partly overlaps the preceding run, and a protective gas atmosphere is maintained above the interaction zone of the laser beam and the metallic powder. Once finished the powder bed is removed from the machine and excess 
Fig. 1 Schematic of the DMLM process [10] (reproduced with the permission of the Verein Deutscher Ingenieure eV, Germany)

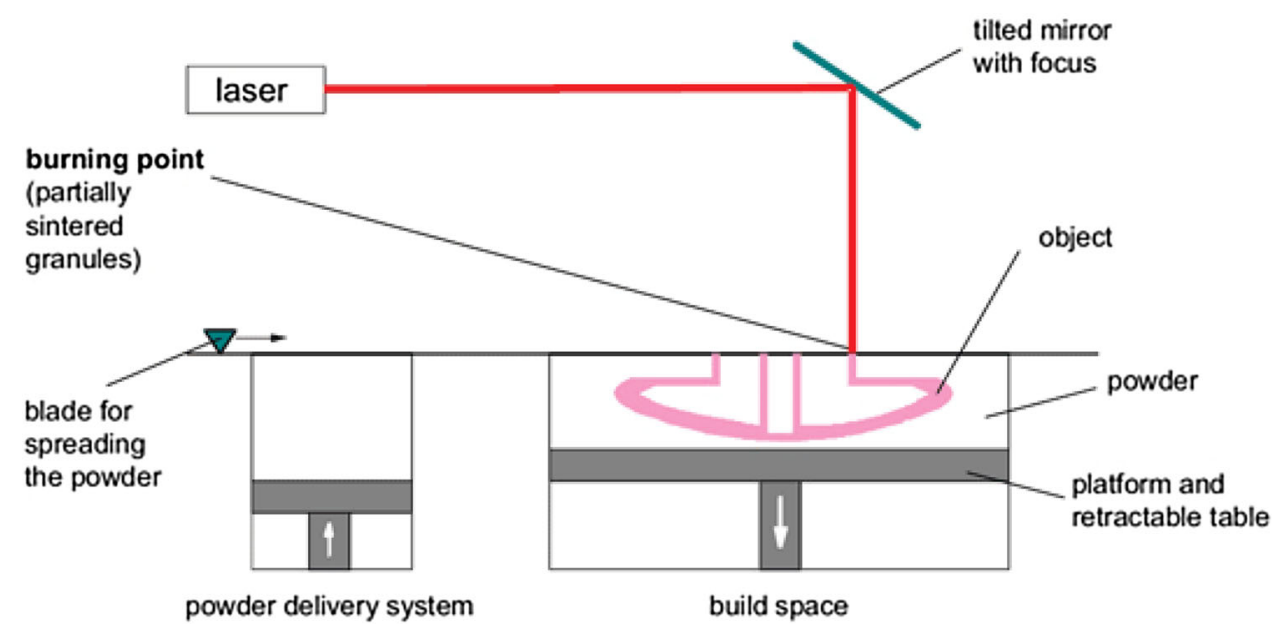

powder is then removed and can be fully recycled, although some sieving of larger particulates may be required. Figure 1 shows a schematic of the process $[12,13]$.

A key opportunity is presented to the EU jewellery manufacturing sector through the harnessing of the emergent and rapidly maturing DMLM technologies and processes, which will facilitate the manufacture of uniquely designed, high-value-added, often custom-made, personalised, jewellery products that will be inherently resistant to being copied [14]. The initial concept and design phases define the innovative nature of a jewellery product during the early stages of its development. The process of design enables the definition and development of concepts and ideas and individual personalisation or customisation of an item, facilitating commercially viable new product development. The transfer of these concepts and ideas into jewellery products is achieved through a variety of technical processes including CAD [15] 3D printing/prototyping [16], and light engineering-based processes and technical feasibility to manufacture is traditionally a vital consideration at each stage of the design process. As previously discussed, consumer demand for increasingly novel products has resulted in the need for extreme flexibility in the design [17] and production of jewellery, the ability to respond rapidly to ever changing demands, and the implementation of a streamlined product development process by manufacturers of personalised and custom-made products, not just jewellery. The EU jewellery industry has a significant global reputation for producing well-designed, well-made, high-quality jewellery products, manufactured in controlled and regulated environments that meet the high expectations of their end consumers. A radically new manufacturing approach could be considered as being useful to help re-energise the precious metal jewellerymanufacturing base and to help facilitate new opportunities to boost production, increase profitability and regain market share. DMLM, which is nowadays routinely considered to be part of the rapid or additive manufacturing (AM) stable of technologies, has now become an accepted production solution within a range of industrial manufacturing sectors including aerospace, automotive, dental and medical, where AM is used to manufacture parts in a range of ceramic, polymeric and base metallic materials.

Early research undertaken in Sweden in 2005 [18] and much more recently in the UK [19] and Europe has demonstrated that DMLM technologies could be extended to the manufacture of products in precious metals, including 18 carat golds in various colours, silver alloys and even platinum group metals [20]. This research led to some early global interest in the use of DMLM for precious metal parts manufacture. However, the principal experience of precious metal DMLM to date has been largely limited to the cosmetic dental industry, which has adopted some digital production solutions in precious metals. In addition, this use of the technology in dentistry was restricted to a small number of specialist gold alloys used for restorative dental crowns in non-jewellery specific alloys. Many potential alternative uses for precious metal DMLM have also been identified including electronics, fuel cell, medical, catalytic and satellite applications and in the manufacture of low-volume, high-value components in the prestige automotive, biomedical and marine sectors [21]. There has also been much discussion concerning the potential for precious metal DMLM and its inherent design benefits within the jewellery and high-value goods sectors, but the research, capital investment, and specialised metallurgical knowledge base required to set up a precious metals DMLM sector have until now been considered largely prohibitive [22]. Currently there are a small number of different DMLM technologies at various stages of development and use in and around the European jewellery 
Fig. 2 Examples of students' work using DMLM technology from the Birmingham School of Jewellery 2014/15 DFI DMLM module. From left to right:

Natalia Antunovity, Suyang Li, Tesni Odonnell, and Tomas

Binkevic
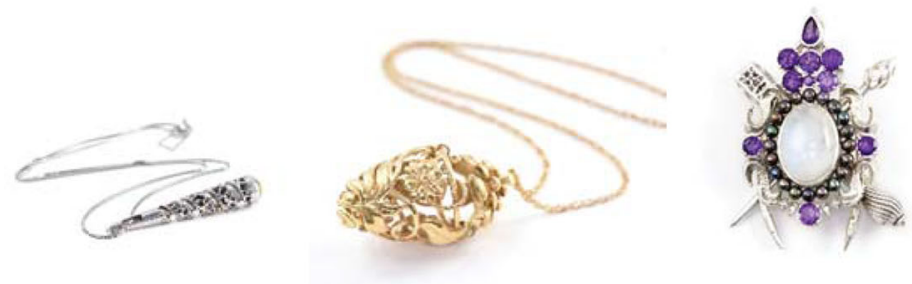

sector. In 2011, the JIIC introduced and continues to deliver a teaching module specifically about DMLM technologies and their adaptation for jewellery design and manufacture to the cohort of the Design for Industry (DFI) students at the Birmingham School of Jewellery. Figure 2 shows just a few examples of the class of 2014/15 work on jewellery designed for the DMLM process. Each of them explores and takes advantage various aspects of the geometric design freedoms that the DMLM process offers. These items were produced for the students in a number of different metals by a UK-based supplier of a DMLM technology and were built on a Concept Laser Mlab LaserCUSING $^{\circledR}$ by ES Technology Ltd.

The ability to successfully introduce this new technology coherently and efficiently into a business, whether it be jewellery design, manufacture, retail or e-commerce [23] is key to the AM supply chain but AM will inevitably provoke change and adaption at the organisational level. For customised or personalised items data collection and design methods will need to be re-assessed and tailored. Depending on the supply chain variant adopted many key decisions will need to be made such as to whether to purchase AM technology and operate it 'in house', or to purchase AM production from a third party supplier [24]. Both options impact on logistics and distribution but quite possibly the largest and to date unknown impact could well be on a company's culture and how, or if, it changes to accommodate AM. Those considering adopting AM principles either fully or in part should also consider the following tactical business strategies and paths as part of their business model or supply chain conundrum. Here we attempt to identify the critical routes to the outcomes sought by those jewellery manufacturing companies thinking of applying an AM technology to their business strategy (e.g., weight reduction, customisation, individualisation, personalisation, coping with fluctuating demand, volumes of one, etc.)

\section{Stasis}

AM can be considered to offer opportunities to deliver improvement to targeted areas of performance in companies independent of any desire to significantly alter the product range or supply chain improvements. Maintaining a stasis of this kind has been the general principle behind the foothold that $\mathrm{CAD}$ and prototyping has gained in the jewellery sector over the past $10-15$ years. It has been used to streamline and accelerate the jewellery design process resulting in reduced time to market, reduced cost and improved quality. In general, performance-enhancing efforts related to maintaining stasis deliver value to the adopting company through cost reduction and acceleration of the product cycle. The result of this can include a reduced time to market, improved product quality, increased product complexity and reduced costs. In general, performance-enhancing efforts related to the Stasis pathway deliver value by improving profitability through cost reduction and by accelerating the speed with which the resulting cash flows can be delivered (by accelerating the business cycle). The use of these early adopted and underpinning AM principles requires neither dramatic supply chain redevelopment nor product redesign to deliver value to the adopter, and offers a low risk starting point from which companies can consider integrating these technologies.

\section{Supply chain evolution}

Similar to the stasis path this route presents many significant opportunities to improve company performance, this time by transforming the supply chain in pursuit of improved company performance and growth. These benefits can be accrued from AM's ability to reduce minimum efficient scale in production locations, alter (considerably shorten) the traditional supply chain and reduce working capital requirements (WIP and stock) and could lead to improved performance to profit (cost,) risk and reduced time to market by giving companies the ability to deliver their products faster and meeting their customers' high expectations. In the long run, such shifts in supply chain structure may represent a key growth vector, as firms large and small try to capitalise on the ability to deliver faster, cheaper, and more precisely than their competitors. 


\section{Product evolution}

This is the path along which companies can begin to be truly innovative by adopting and adapting their business model to the principles of AM by the creation of unique products that cannot be produced by other, more traditional means. Customised, personalised and individualised products, should flourish along this path as the early adopters exploit their ability to quickly meet, or even create, their customers demand for specialist and unique products. In addition the ability to closely monitor the build process in ways that are not possible using other traditional jewellery manufacturing methods presents opportunities to enhance company performance by the mitigation of risk and improved delivery performance.

\section{Combined product and supply chain evolution}

This route encourages companies to consider either sequential or simultaneous transformations of both their products and the supply chains that deliver both their unique products and the desired business growth. In essence, a combination of the two that seeks to obtain not only the operational benefits but to create new markets that impair the non-AM competitor's ability to compete or copy. For instance direct interactions with the customer in the design process, the co-design principle, will result in a custom-made product for which the customer will expect to pay a premium price. It is also reasonable to speculate that the route to this pathway runs through the product innovation goals that characterise the product evolution route. The delivery of innovative products may require new or revised approaches to supply chains and distribution, or it may present opportunities to disrupt competitors and markets when combined with supply chain innovation. This may be particularly true where AM and other digital technologies are arranged to increase the level of collaboration between producers and end users. Non-AM aspects of the production process (e.g., setting stones into a piece of jewellery) may determine where physical production is likely to take place, but disintermediation (removal of intermediaries) may turn out to be a feature of the resulting supply chains. AM represents an innovative technology and as a result companies are presented with intriguing choices about how to extract available value from the AM process.

The JIIC is also currently actively involved with a UK government funded DMLM of precious metals research scheme called the precious project, whose mission statement reads:

"To demonstrate the viability of precious metal additive manufacturing within the UK Jewellery industry from design and manufacturing through to finishing, polishing and retail" [25].
This is a 2-year project aimed at elevating the current state of the art of DMLM AM within the UK precious metal jewellery industry. After a piece of jewellery has been designed and before it can be manufactured using DMLM a small number of core activities need to be both clearly understood and to take place:

- Pre-processing (preparing pieces for manufacture). This essentially refers to all front end software-related activities including the design process.

- Processing (manufacturing jewellery items using $D M L M)$. This refers to the actual manufacturing process using DMLM technologies.

- Post-processing (manual and automated finishing and polishing processes). This refers to the post-DMLM manufacture processing stages up to the point where an article is ready for sale. Each of these steps is interdependent on the others and they must take place in a logical sequence. When combined together effectively this can result in the production of novel and unique quality jewellery items. Understanding these various activity interrelationships has a profound effect on the eventual quality of any DMLM printed jewellery. If a jewellery designer understands, even on a fairly superficial level, what is involved in each of these core activities then they will be able to better design jewellery that not only takes advantage of the geometric freedoms that DMLM offers but also can be suitably post-processed to an acceptable quality of finish.

One of the most important pre-processing steps is the creation of the support structures [26]. Because the processes of DMLM requires the jewellery design to be virtually 'sliced' in the machines own software (much like a loaf can be sliced up only these slices are much, much thinner.) The parts are built up of multiple slices printed by the AM technology one on top of the other. Metal parts being built using AM require a support structure, this is a "scaffold" like construction, and which supports all over hanging parts enabling under cuts, voids and holes to be produced. A jewellery designer will not need to be able to create the supports, as they are added by the DMLM machine operator and the technologies operating software package, but they will need an appreciation of the use and application of supports as they leave a witness mark/scaring when they have been removed, which will require cleaning up and finishing (similar to that required when removing a casting sprue). In theory, it is possible to build any shape, however, if supported areas are visible but inaccessible, then the result will be perceivably poor surface quality. A small change to a design can eliminate the need for a lot of support structures. Support structures are required in most, if not all, laser-powered, metal-based AM processes, and there are a number of divergent reasons for 
their presence. To build complex geometries with overhanging and undercut surfaces, support structures are required to assist in controlling defects such as curling, sagging, cracks, shrinkage and/or other deformations of the part being built. These defects may be caused by the typical thermal stresses of the DMLM process, occasionally by overheating, or most commonly by being dragged over and disturbed by the re-coater or screed blade applying the next layer of powder. But supports are principally required because the powder bed surrounding the melt pool created by the laser is not sufficient to support the liquid metal in place during the printing (melting) process (Fig. 3).

Other functions of supports are bonding the part to the build plate and providing a thermally conductive connection between the part and the build plate to rapidly and effectively dissipate heat from the melt zone. The needs discussed above must be balanced with the cost when considering the design and placement of support structures. Supports can be solid, hollow or even cellular structures that are removed after the build/print cycle has been completed. Thus, they can potentially represent a considerable waste in terms of expensive precious metal material, energy consumption and the time required for their creation, placement, construction and eventual removal (Fig. 4). From a cost standpoint, supports should have as low a volume as possible, using the minimal amount of valuable materials, and be relatively easy to remove.

These almost contradictory needs of strength and thermal conductivity versus the cost to build and remove make automated solutions for creating supports very challenging. Support structures and their placement also have an important role in the developing 'design rules' that are now being more widely understood and applied when designing for the DMLM AM processes. Additive manufacturing processes do not totally eliminate design constraints; they are just different when compared to the more conventional manufacturing processes. For example, while you do not need to worry about draft angles, AM processes have their own limitations. A basic understanding of how part orientation can improve part accuracy and surface finish as well as reduce the production time and volume of support structures required for building a part is important. Many of the earliest studies and research into support structures used in the various industrial AM technologies, particularly those used in stereolithography and Fused Deposition Modelling, have concentrated on the non-metallic processes. These support structures and their creation software programs then went on to form the basis for the development of the support structures used in the various DMLM processes. Being able to use software that can automatically analyse a part with the aim of reducing the amount of support needed to build it is highly desirable

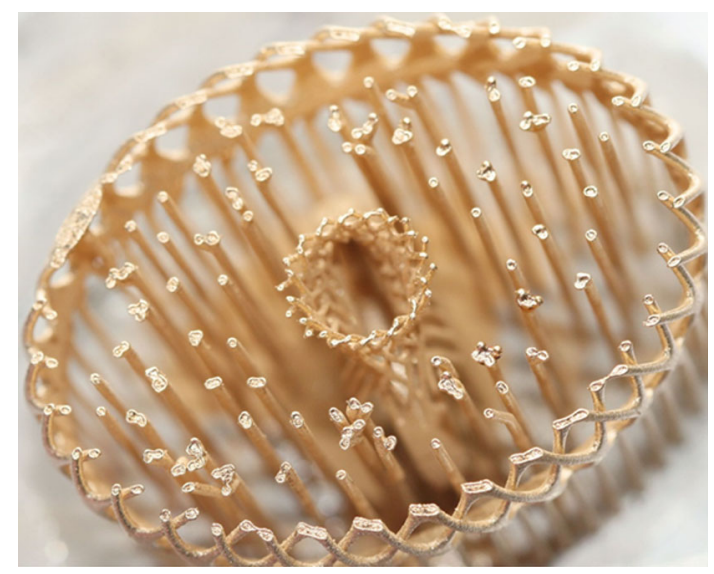

Fig. 3 A build that has been stopped part way through the process and the evidence of the 'melt pools' can be clearly seen at the tips of the strands

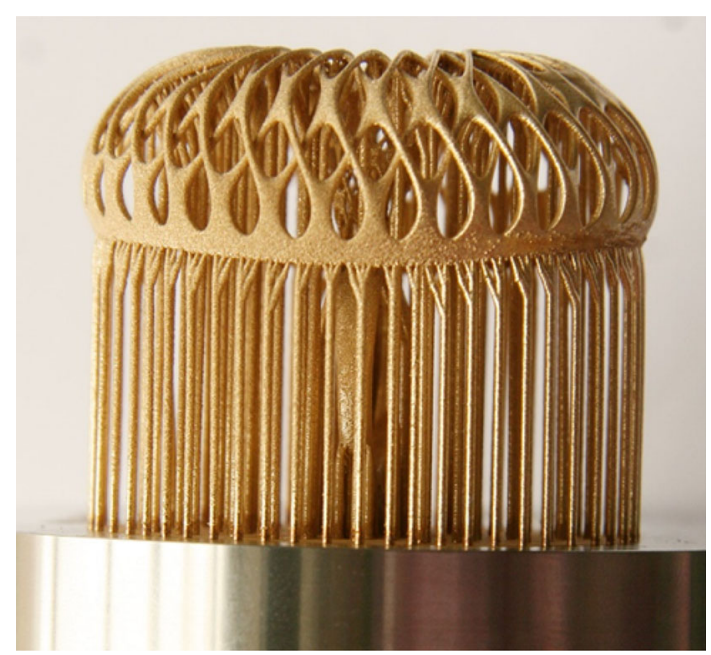

Fig. 4 The same build when finished showing all the support structures in place

as long as the as-built surface finish is not compromised, and the time to build the part does not become uneconomical by selecting an orientation that substantially increases the build height. A number of such new software's are currently under development though not yet generally yet widely available and still in alpha and beta testing, these software's provide quick and easy methods to prepare for production CAD files from both native and neutral formats.

There are four main steps in model preparation for the DMLM process:

1. Checking the quality of the CAD model and repairing any problems it might have.

2. Positioning the part in the optimum orientation for layer-by-layer DMLM manufacture. 
3. Addition of any fixtures, supports and other geometry that might be required to ensure reliable manufacture and easier post-processing.

4. Exporting the complete data in a suitable file format for manufacture.

First, common problems such as gaps, holes, or overlapping faces in the data for both solid and mesh models need to be identified and repaired to give a water-tight CAD model that will be used to produce an accurate and successful part.

Second, orientating the model on the build platform must consider a number of factors, including production time and cost, fixturing quantity, heat effects and surface quality critical areas of the part. The software's should allow the user to try a variety of positions manually or to use built-in orientation strategies with appropriate metrics either for a single part or for a group of parts that will be built simultaneously.

Thirdly, fixtures or supports can be created in the software's to fulfil one or more needs depending on the shape, size and material of the part being processed. They need to be able to analyse the model to identify critical areas that need supports to ensure geometric stability during the build and adequate heat sink management, and to generate the correct fixture/support geometries required. As with the part orientation, the user requires the ability to edit the suggested results manually if required. The positioning of the fixtures also needs to take account of their removal after the build, both to ease their removal and to minimise any witness marks or scars that may be left on the part. In addition to fixtures, other geometry types may need to be added, such as spigots to grip the part during polishing and other finishing operations, or datum tabs to provide a known location for finish machining programs.

The fourth and final stage is the export of the complete model from the software in a suitable data file format for the DMLM equipment. Software's will need to be able to output models in a growing range of manufacturers' proprietary file formats, as well as STL mesh data that can be processed directly by most additive machines' own operating software. As a core activity in AM, creating and working with support structures is a necessary requirement best automated if possible. Below are some examples of the support structures created with the PartBuilder software as well as the actual parts as-built (Figs. 5, 6).

\section{Case study: the Ojo project}

To help illustrate the various DMLM manufacturing steps a pendant piece from the precious project, called 'the Ojo', will be used to show the various stages of designing and manufacturing jewellery for the DMLM process. The Ojo is intended to be an iterative design series of 100 pendants where each pendant produced is significantly different from the pendant before and the pendant after. This is achieved by the use of a CAD design algorithm that continually morphs the basic pendant design. This pendant was conceived and designed by Lionel T. Dean of future factories who is a member of the precious consortium of companies. The CAD file is created, saved as a stereolithography (STL) file and is shown in Fig. 7.

The next step is to use a suitable software to generate and place the support structures, as shown above, that are a necessary part of the DMLM process which requires the jewellery design to be 'sliced' in the software. The parts are built up of multiples of these slices printed by the DMLM technology one on top of the other. Parts being built using DMLM require a support structure, this is a scaffold-like construction, and supports all overhanging parts enabling undercuts, voids and holes to be produced. A jewellery designer will not need to be able to create these supports, as they are added by the DMLM machine setter and the machine's software package, but they will need an appreciation of the use and application of supports as they leave a witness mark or scarring when they have been removed, which will require extra cleaning up and finishing (similar to that required when removing a casting sprue). In theory it is possible to build any shape, however, if supported areas are visible but inaccessible, then the result will be perceivably poor surface quality. A small change to a design can eliminate the need for a lot of support structures. Support structures are required in most, if not all, laser-powered, metal-based DMLM processes, and there are a number of divergent reasons for their presence. To build complex geometries with overhanging and undercut surfaces, support structures are required to assist in controlling any potential defects in or on the part being built. These defects may be caused by the typical thermal stresses of the DMLM process, occasionally by overheating, or most commonly by being dragged over and disturbed by the re-coater blade applying the next layer of powder. Supports are most principally required because the powder bed surrounding the very small melt pool created by the laser is not sufficient to support the liquid metal phase in place. Other functions of supports are the bonding of the part to the build plate and providing a thermally conductive connection between the part and the build plate to rapidly and effectively dissipate heat from the melt zone.

The next step is to fill the machine with powder, [27, 28] load up the STL file and set it into motion. A re-coater blade, or brush, pushes fine, powdered, gold build material from a carefully measured powder supply hopper to create a uniform layer over the build platform. The laser scanning system literally draws the two-dimensional (2D) cross- 
Fig. 5 Examples of automatic software generated support structures
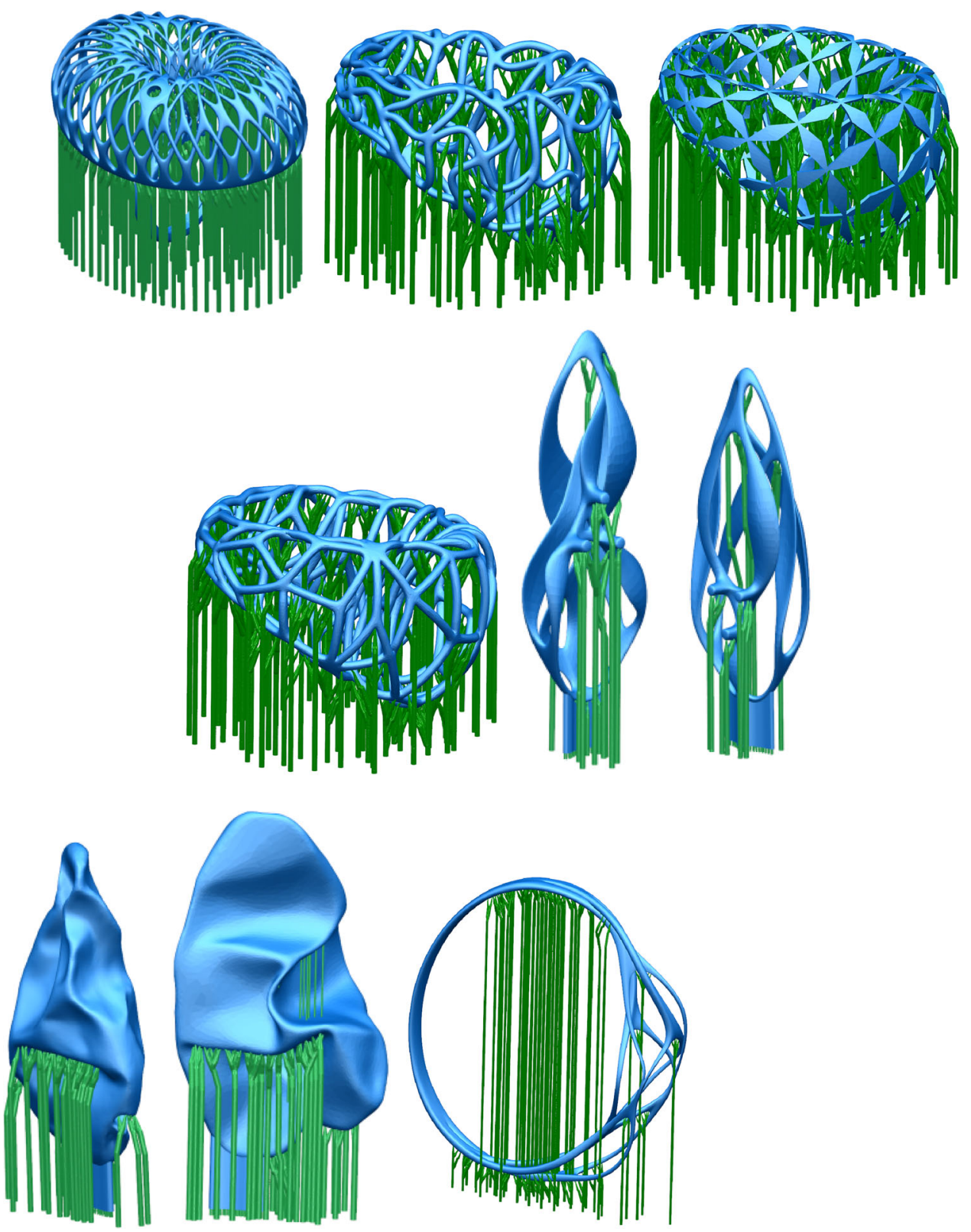

section of the CAD file slice on the surface of the gold powder build material, melting it into a solid deposit layer or slice as shown in Fig. 8 [29, 30].

After the first layer is produced, the piston beneath the build platform is lowered fractionally and another powder layer is pushed into place using the re-coater blade. The laser beam melts the second layer and at the same time fuses or bonds it to the layer below. This process is repeated layer-by-layer until the part is completed. It is this layer adding process that leads to this technology being described as 'additive manufacturing' or 'additive layer manufacturing' in many quarters [31].

Once the build process has been completed the build plate piston is slowly raised, the surplus unused powder is carefully swept away and the 'additively manufactured' jewellery item is exposed (Fig. 9).

The part is then removed from the build platform and the support structures are also removed (Fig. 10).

The witness marks or scarring left by the supports also have to be removed in much the same way as a casting sprue has to be removed and cleaned up. Because the support structures are much smaller than a typical casting sprue, a small, fine, burr on a pendant or Dremmel drill will often suffice. Once the supports have been removed the parts are then ready for finishing and polishing (Fig. 11).

Mechanical, or mass finishing, techniques are often found to be the best for this stage because the DMLM of 
Fig. 6 Examples of various asbuilt platforms showing the actual supports printed from the CAD data generated by the automatic support generation software

Fig. 7 CAD design and showing support structures for the Ojo
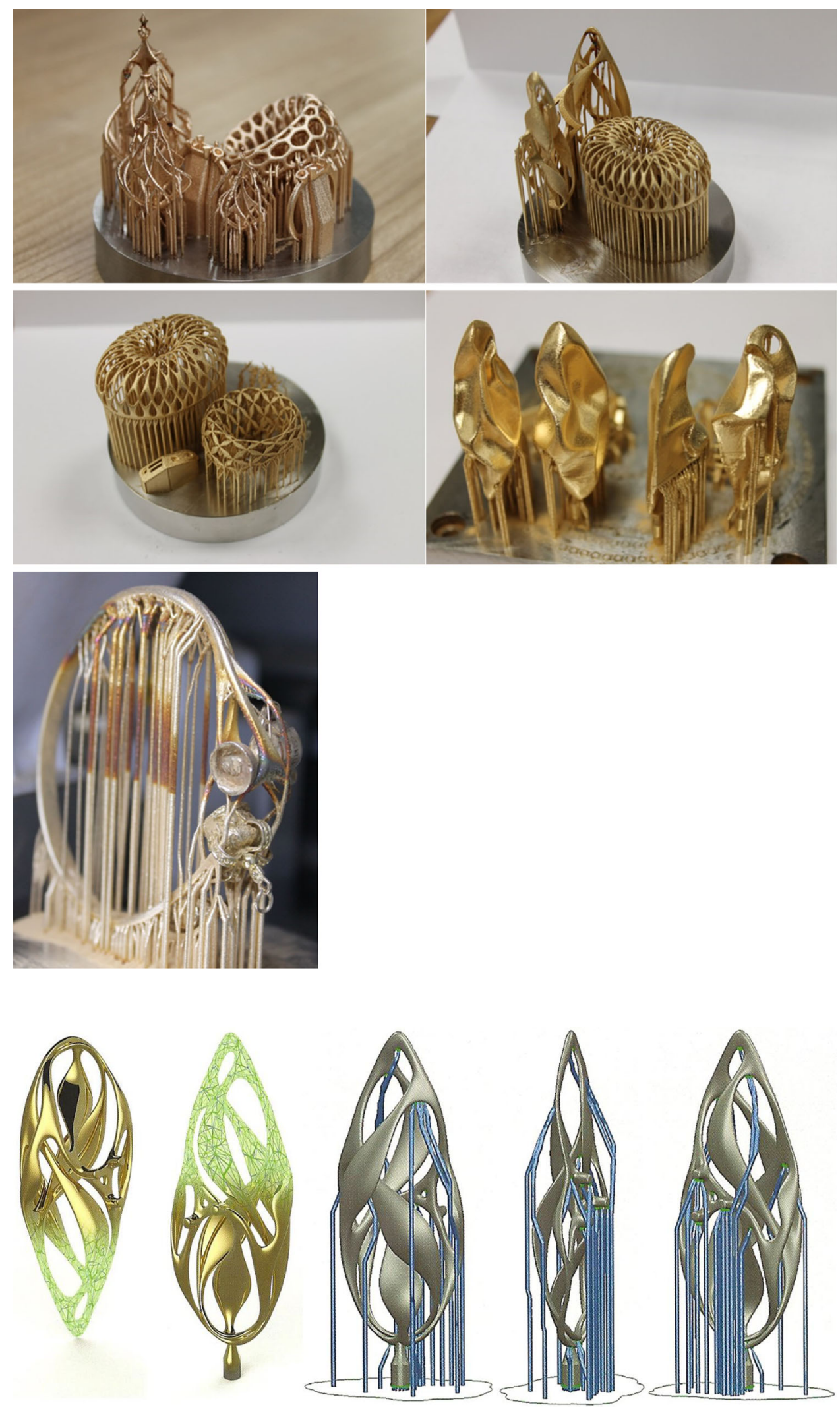


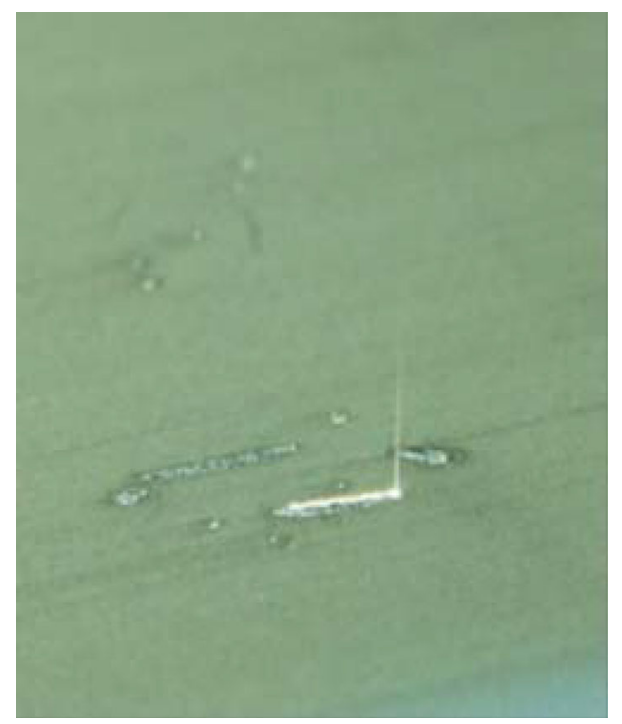

Fig. 8 The laser beam observed as it scans across the surface of the thinly spread gold powder

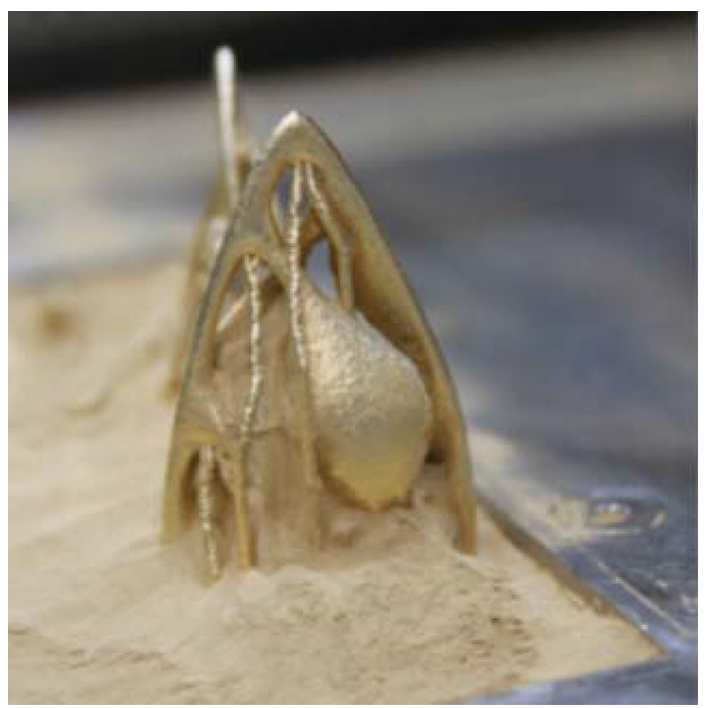

Fig. 9 An 'Ojo' seen emerging from the bed of gold powder

jewellery will probably prove to be most commercially effective when used to produce geometrically complex designs. These designs by their very nature will present many unique and product specific challenges when ready to be polished and finished. Mass finishing technologies are based on the correct application of media flow pressure and speed to the jewellery item to be polished. Generally, the higher the pressure exerted by the media on the jewellery, and the faster the media flows across the jewellery parts, the faster the desired finishing results can be achieved. But this flow has to be either uniform or directed, depending where the polishing is required. Centrifugal disc finishing is an industrial mass finishing process adapted for the

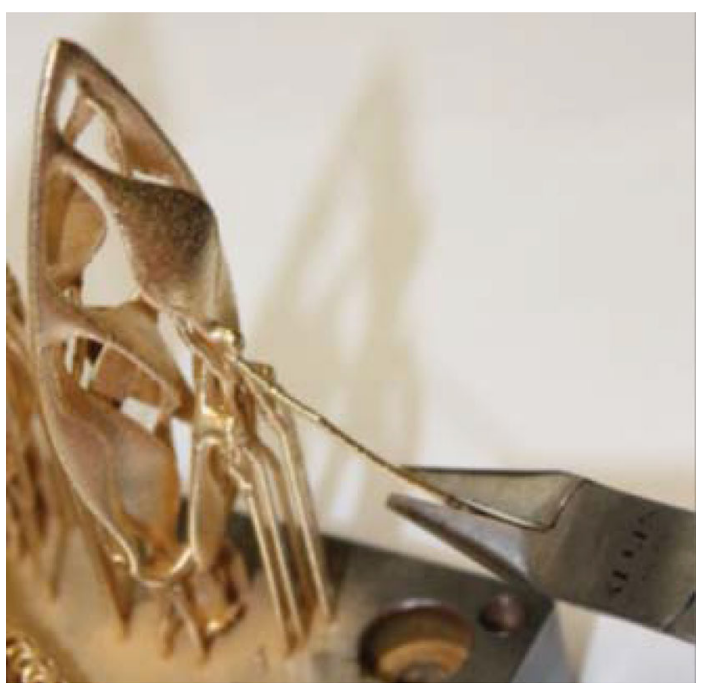

Fig. 10 Support removal using pliers
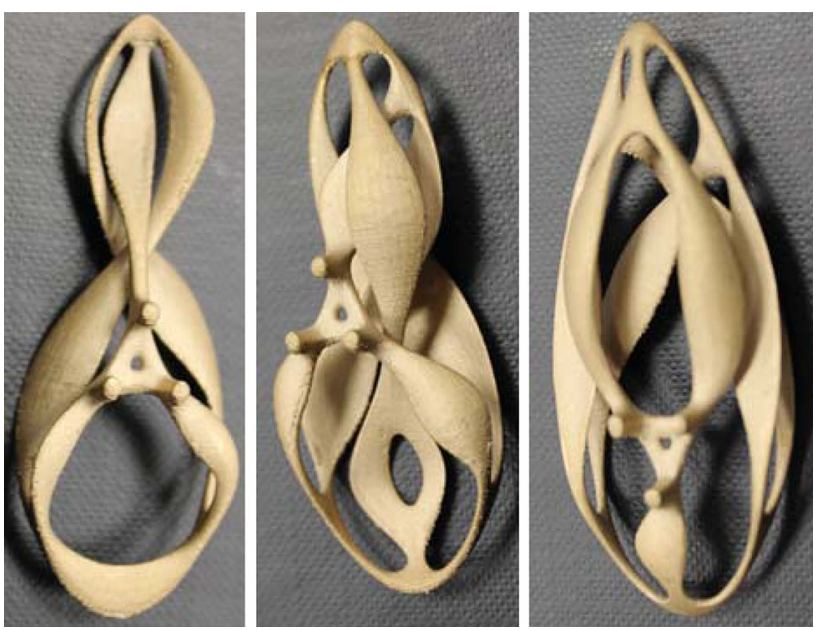

Fig. 11 Three variants of the Ojo shown prior to mechanical and manual finishing and polishing

surface treatment of jewellery. The process is carried out in a cylindrical container which is open at the top, while the bottom consists of a turntable-like disc separated from the container wall by a microscopically small gap. During operation, the work pieces and the grinding or polishing media in which they are immersed rotate at a high speed, creating a toroidal abrasive flow of the media; the relative difference in speed of the components and media produces the polishing effect. The contact between the jewellery pieces and the medium generates a very intense finishing effect which is up to 20 times more efficient than can be achieved with conventional systems like vibratory finishers. A process refined by precious consortium partner Finishing Techniques Ltd is the 'stream finishing' process (sometimes referred to as immersion polishing). This is a fairly new concept to jewellery polishing and features short 
processing times because the medium is compressed against the wall of a large spinning bowl (centrifugal disc finishing) and the parts are held and rotated in this flow by use of a rotating fixture similar to an electric drill chuck on an extended shaft. Because the rotating head is fixed but with an adjustable angle of attack when immersed into the bowl, it can be easily automated and has shown excellent reliability and repeatability (Figs. 12, 13, 14, 15, 16, 17). The use of small, light media can produce an excellent finish, the finishing energy coming from the relative speed of both the jewellery part and the medium. A final polish by hand completes the process.

\section{Comparison with casting}

The most widely used technology in jewellery manufacturing worldwide continues to be the lost-wax investment casting process, accounting for an estimated $80 \%$ of all jewellery production. Current investment casting processes have been highly developed over time principally to facilitate traditional historical demands for high-volume, batch- and mass-produced jewellery products. In contrast, there is an emerging need for a low-volume, rapid response to consumer demands for custom-made, individually designed products, in simple terms producing in volumes of one. DMLM could meet this need. Additionally, yet more pragmatically, it also offers the potential for creating items that appear solid yet, if sectioned through, would prove to be entirely hollow or contain a simple honeycomb or scaffolding structure added for strength, a process sometimes described as 'volume without mass'. Such forms and designs are not currently achievable using the traditional jewellery manufacturing processes. Caution must be applied, of course, in selecting appropriate items for this type of manufacture. For instance making a typical wedding band hollow would result in an item that feels valueless and cheap; whereas a bulky, heavy, watch bezel
Fig. 12 Examples of typical centrifugal disc finishing equipment

Fig. 13 Example of stream finishing equipment

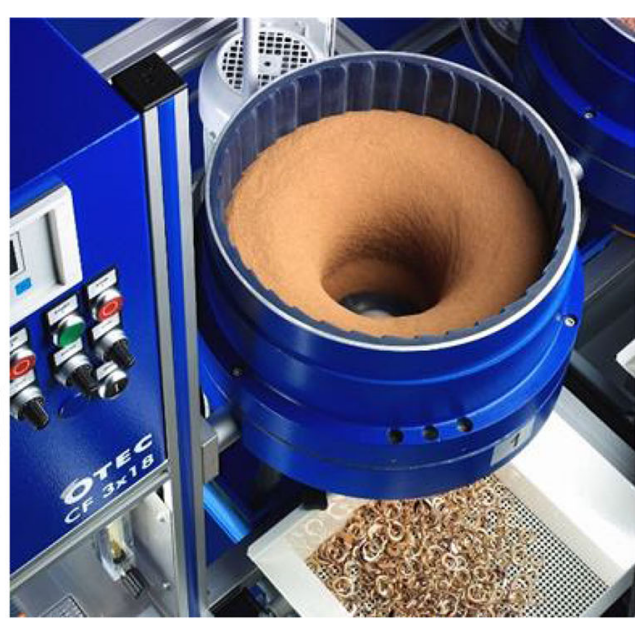

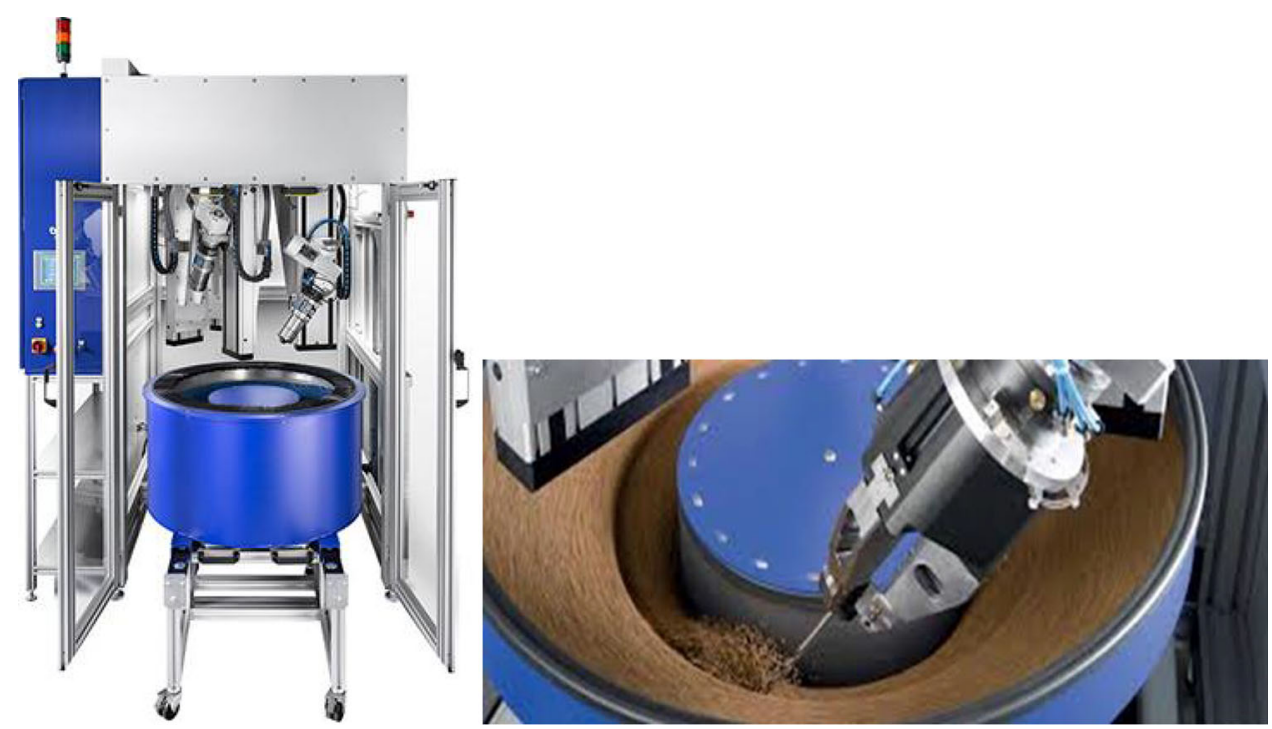


Fig. 14 An example of an Ojo being stream finished
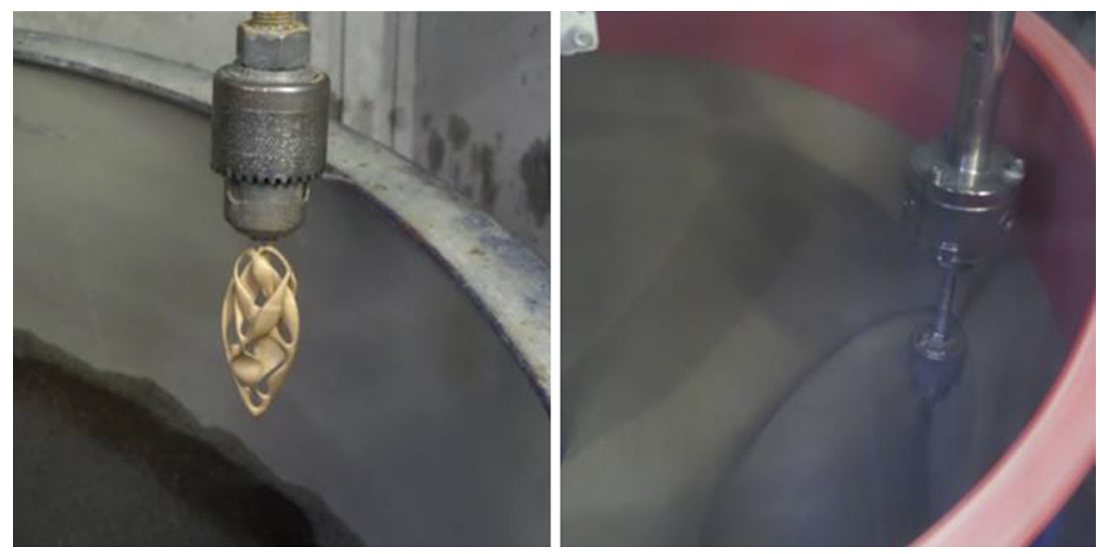

Fig. 15 An Ojo after $30 \mathrm{~min}$ stream finishing and $1 \mathrm{~h}$ stream finishing
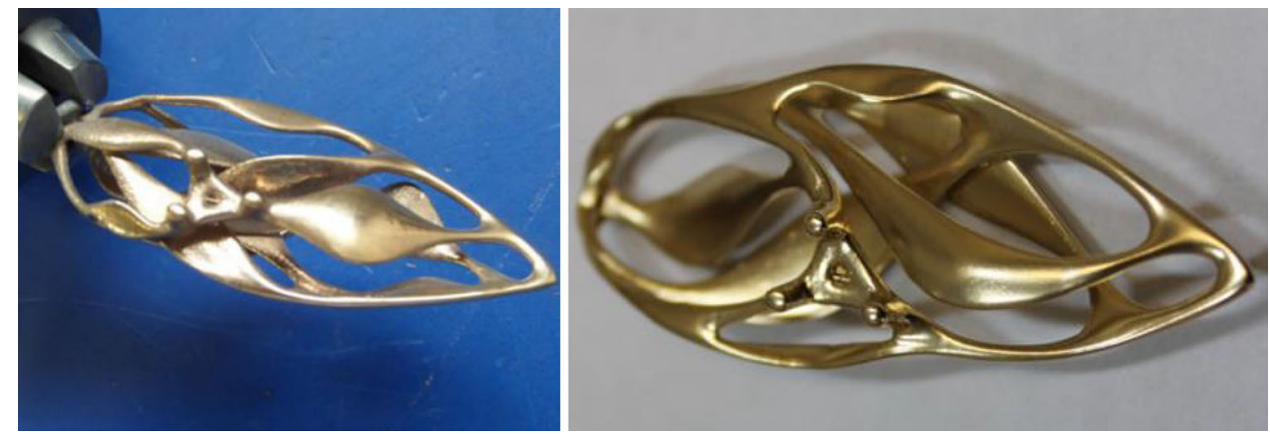

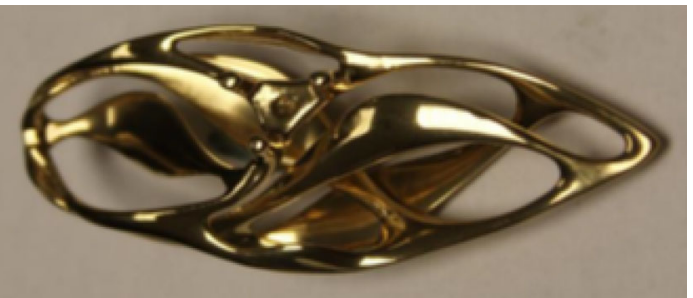

Fig. 16 An Ojo that has also been hand polished after $1 \mathrm{~h}$ stream finishing

could be produced using a weight reducing hollow profile and the resultant watch would still feel right, with weight being added by the watch movement and wrist band (Fig. 18).

The potential savings in the intrinsic cost of expensive raw materials is undoubtedly now approaching the point at which this novel manufacturing process can become more commercially viable and attractive to the jewellery manufacturer and the consumer. Furthermore, it is entirely possible and correct to consider at this point that the widespread supply chain model used in jewellery manufacturing throughout the EU, especially in relation to lostwax investment casting-namely the use of sub-contract bureau service providers-is equally applicable to smaller jewellery designers and manufacturers accessing the

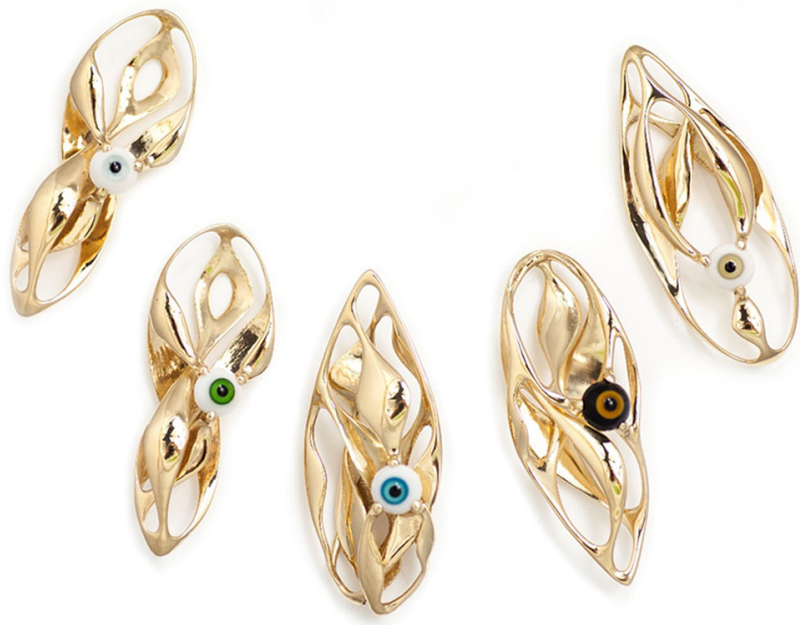

Fig. 17 The end result of the mechanically and hand polished and then stone set Ojo series (Ojo is Spanish for eye!)

DMLM technological advance. In conventional jewellery manufacturing, there is a measurable correlation between part complexity and its manufactured cost. Using DMLM means that not only is complexity independent of tooling costs, but also that virtually any geometry conceived by the designer is theoretically possible to produce. Conventional design methods are based on the 'design for manufacture' 
Fig. 18 'Volume without mass' a hollow wedding band and before and after polishing hollow watch bezels
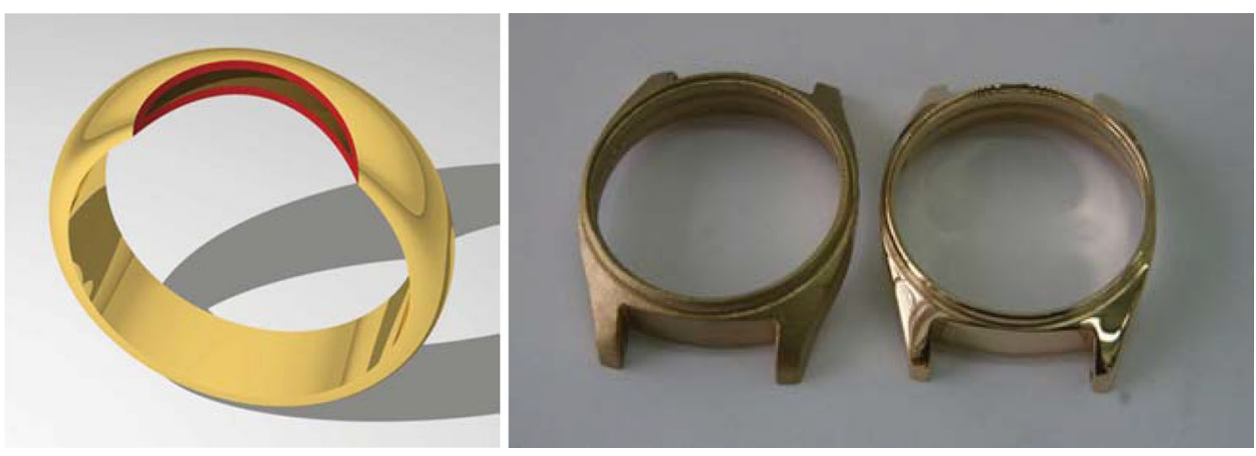

Fig. 19 A Cooksongold Precious M080 owned by the School of Jewellery and an 18 carat gold galleon printed on it

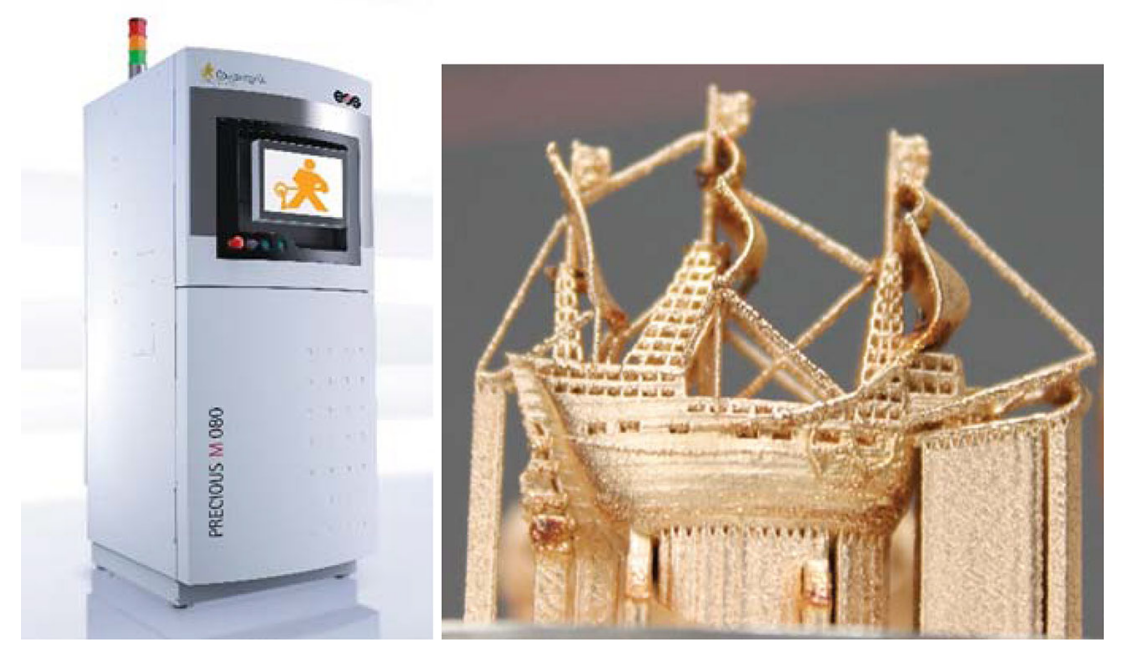

principle, in which manufacturing constraints are included at the earliest stages of the design process. This often results in modular designs with standardised components, meaning designers inevitably modify their design intent to enable the item to be manufactured using a specific manufacturing process. Using DMLM would allow the removal of many of these constraints, although (as in any other manufacturing process) DMLM has its own limitations. It will be necessary to develop specific 'design rules' expertise to manage and optimise these new and exciting possibilities. Current research at the JIIC in conjunction with the School of Jewellery and its students is based around discovering these rules, which will minimise the limitations imposed by machine modifications and CAD design adjustments, to attain the objective of being able to produce the widest possible range of geometries. The Jewellery Industry Innovation Centre has recently purchased a Cooksongold Precious M080 direct metal '3D printing' DMLM machine for teaching and research purposes, and the image shows an example of one of the Centre's early explorations of the machine's capabilities. The ship is a little over $1.5 \mathrm{~cm}$ high (Fig. 19).

\section{Conclusions}

Manufacturers are generally limited in their methods of fabrication by the cost of tooling, which must be amortised over the number of parts produced during the life cycle of a tooling product. In existing conventional jewellery manufacturing, there is a direct link between the complexity of the part and its manufactured cost; this can be significantly reduced with use of the DMLM processes. In gaining knowledge and understanding of the potential design and manufacturing advantages of DMLM, jewellery manufacturers should be provided with the economic impetus to consider adopting DMLM processes, as appropriate to their company's needs. Many high-value products are made in small volumes or require individual, personalised adaptations for each customer or application. The ability to provide such modifications, as well as the availability of toolless fabrication, will influence what is designed, how it is designed, and the quantity of products offered [32]. Innovative design could be considered vital for the survival of the high-value-added industries, including jewellery manufacturing, though it should be remembered that the 
manufacture of well-designed unique products remains a labour intensive, expensive, and consumer-centred process. The commercial pressures to reduce costs to remain competitive, while retaining design quality, challenge jewellery manufacturers to find ever more innovative manufacturing techniques as well as consider alternative routes to their markets and consumers. The jewellery industry and other design-led creative industries are ideally suited for developing the new interfaces between the customer and designers, and they will also need to consider new production technology approaches that maintain and exploit this competitive edge. Jewellery is therefore in a unique position to capitalise and further develop the potential of DMLM, while using generic fabrication criteria which are relevant to many other high-value-added industries where custom-made products command correspondingly higher consumer prices.

\section{References}

1. Krijger M (2014) 'CBI trade statistics for jewellery', global intelligence alliance/centre for the promotion of imports from developing countries (CBI). Ministry of Foreign Affairs, The Hague

2. Growth Dedicated (2002) Call 10/00', topic IV 31, The European Virtual Institute For Jewellery Technology, EC funded project, reference G7RT-CT-2001-05065. Italy, Milan

3. Street L, Gopaul K, Kumar M, Lu C, Hewitt A (2015) Gold demand trends: first quarter 2015. World Gold Council, London

4. Krijger M (2015) 'CBI trends: jewellery', global intelligence alliance/centre for the promotion of imports from developing countries (CBI). Ministry of Foreign Affairs, The Hague

5. Penfold DG (2007) 'New product development in the West Midlands region of the UK jewellery manufacturing industryevaluating the impact of design support'. In: Bell E (ed) The Santa Fe Symposium on jewellery Manufacturing Technology 2007. Met-Chem Research, Albuquerque, New Mexico, USA, pp 445-456

6. Jewellery \& Watches (2014) Key note market report. Key Note Ltd, Richmond on Thames

7. Penfold DG (2007) Design J 10(1):3

8. M. Karydes (2015) 'Bold gold jewelry is back in style', Fortune, 9th May

9. Wiesner K (2005) Sintering technology for jewellery and multicolour rings. In: Bell E (ed) The Santa Fe Symposium on Jewelry Manufacturing Technology 2005. Met-Chem Research, Albuquerque, New Mexico, USA, pp 501-520

10. Meiners W, Wissenbach K, Gasser A (1998) Fraunhofer Ges Forschung, Germany, 'shaped body especially prototype or replacement part production. German Patent 19:649-865

11. Patrick S (2005) CAD software for jewelry design: a comprehensive survey. In: Bell E (ed) The Santa Fe Symposium on Jewelry Manufacturing Technology 2005. Met-Chem Research, Albuquerque, New Mexico, USA, pp 409-422

12. VDI-Richtlinie: VDI3404GenerativeFertigungsverfahrenRapid-Technologien (Rapid Prototyping)—Grundlagen, Begriffe, Qualitätskenngrößen, Liefervereinbarungen' (Engl. Transl. 'VDI Guideline: VDI 3404 Additive fabrication-Rapid Technologies (Rapid Prototyping)—Fundamentals, Terms, Quality Parameters,
Supply Agreements'), December 2009 (Withdrawn December 2014), Verein Deutscher Ingenieure eV, Düsseldorf, Germany, 2009

13. Fischer-Buehner $\mathbf{J}$ et al (2012) Rapid jewelry manufacturing by laser melting of precious metal powders (PLM): fiction or future? In: Bell E (ed) The Santa Fe Symposium on Jewelry Manufacturing Technology 2012. Met-Chem Research, Albuquerque, New Mexico, USA, pp 177-202

14. Carey AM (2010) The changing demands on the creative process as a consequence of new technologies. In: Bell E (ed) The Santa Fe Symposium on jewellery Manufacturing Technology 2010. Met-Chem Research, Albuquerque, New Mexico, USA, pp 101-118

15. Adler S, Fryé T (2005) The revolution of CAD/CAM in the casting of fine jewelry. In: Bell E (ed) "The Santa Fe Symposium on Jewelry Manufacturing Technology 2005. Met-Chem Research, Albuquerque, New Mexico, USA, pp 1-24

16. Wiesner Klaus (2005) Sintering technology for jewellery and multicolour rings. In: Bell E (ed) The Santa Fe Symposium on Jewelry Manufacturing Technology 2005. Met-Chem Research, Albuquerque, New Mexico, USA, pp 501-520

17. Penfold Gay (2008) Designing for rapid manufacturing and other emerging technologies. In: Bell E (ed) The Santa Fe Symposium on Jewelry Manufacturing Technology 2008. Met-Chem Research, Albuquerque, New Mexico, USA, pp 243-256

18. Towe N (2006) 'Laser sintering process for making hollow jewellery', jewellery technology forum proceedings, June 2006. Legor Group, Vicenza, Italy

19. Khan M, Dickens P (2010) Gold Bull 43(2):114

20. Hancox AL, McDaniel JA (2009) Int J Powder Metall 45(5):43

21. Bertol LS, Júnior WK, da Silva FP, Aumund-Kopp C (2010) Mater Design 31(8):3982

22. Simchi A (2006) Mater Sci Eng A 428(1-2):148

23. Fletcher D, Staniorski A (2014) Use of eManufacturing Design Software and DMLS in the Jewelry Industry. In: Bell E (ed) The Santa Fe Symposium on Jewelry Manufacturing Technology 2014. Met-Chem Research, Albuquerque, New Mexico, USA, pp 155-170

24. Cooper F (2012) Sintering and additive manufacturing: the new paradigm for the jewelry manufacturer. In: Bell E (ed) The Santa Fe Symposium on Jewelry Manufacturing Technology 2012. Met-Chem Research, Albuquerque, New Mexico, USA, pp 103-122

25. Precious, Innovate UK, The Technology Strategy Board, Swindon, UK. http://www.precious-project.co.uk/. Accessed October 2013

26. Cooper F (2014) 'DMLM supports: are they the Jewellery Industry's New Sprue, Riser and Gate Feed?'. In: Bell E (ed) "The Santa Fe Symposium on jewellery Manufacturing Technology 2014. Met-Chem Research, Albuquerque, New Mexico, USA, pp 89-109

27. Strauss JT (2008) Powder metallurgy in jewelry manufacturing: status report and discussion. In: Bell E (ed) The Santa Fe Symposium on Jewelry Manufacturing Technology 2008. Met-Chem Research, Albuquerque, New Mexico, USA, pp 295-306

28. Strauss JT (2009) Rapid manufacturing (RM) and precious metals. In: Bell E (ed) The Santa Fe Symposium on Jewelry Manufacturing Technology 2009. Met-Chem Research, Albuquerque, New Mexico, USA, pp 395-416

29. Zito D et al (2012) Latest developments in selective laser melting production of gold jewelry. In: Bell E (ed) The Santa Fe Symposium on Jewelry Manufacturing Technology 2012. Met-Chem Research, Albuquerque, New Mexico, USA, pp 537-562

30. Zito D et al (2015) Definition and solidity of gold and platinum jewelry produced using selective laser melting ( $\left.\mathrm{SLM}^{\mathrm{TM}}\right)$ technology. In: Bell E (ed) The Santa Fe Symposium on Jewelry 
Manufacturing Technology 2015. Met-Chem Research, Albuquerque, New Mexico, USA, pp 455-492

31. Zito D et al (2014) Optimization of SLM technology main parameters in the production of gold and platinum jewelry. In: Bell E (ed) The Santa Fe Symposium on Jewelry Manufacturing Technology 2014. Met-Chem Research, Albuquerque, New Mexico, USA, pp 439-470
32. Lionel Dean T (2015) Precious: another layer of luxury. In: Bell E (ed) The Santa Fe Symposium on Jewelry Manufacturing Technology 2015. Met-Chem Research, Albuquerque, New Mexico, USA, pp 67-80 